\title{
What Does the Strongest Meaning Hypothesis Mean?
}

\author{
Yoad Winter \\ OTS, Utrecht University
}

\section{Introduction}

The variety of interpretations exhibited by sentences with reciprocals is a longstanding challenge for semantic and pragmatic theories. This puzzle is related to some hard questions about the interpretation of plural predicates in general. In this paper we study the implications of relations between reciprocity and plurality for the account of reciprocals in Dalrymple et al. (1994). It will be proposed that Dalrymple et al.'s Strongest Meaning Hypothesis about the interpretation of reciprocals has a more general status in the semantics of plurality. The extended principle proposed determines the interpretation of a plural predicate according to lexical semantic properties of the corresponding singular predicate. The reformulation of Dalrymple et al.'s hypothesis will be shown to derive correct predictions about the interpretation of plural predicate conjunction and transitive plural predicates. The extension of the strongest meaning hypothesis also eliminates the ambiguity of reciprocals in Dalrymple et al.'s proposal and employs the standard Boolean analysis of conjunction. The relevant problems associated with the interpretation of plurals are not conceived as formal semantic phenomena proper. Rather, the strongest meaning hypothesis is formulated as a principle that non-standardly changes formal meaning using lexical semantic knowledge on predicates.

\section{Dalrymple et al.'s proposal}

One case that exemplifies the motivation for the strongest meaning hypothesis is the contrast between sentences (1a) and (2a). Assume that the girls in both cases are Mary, Sue and Jane. (1a) and (1b) are contradictory. However, (2a) and (2b) are not: they are both true in case Jane is standing on Sue, who is in turn standing on Mary. Tentatively, we conclude that (1a) is equivalent to (1c) (but see some qualifications below) while (2a) is not equivalent to (2c).

(1) a. The girls know each other

b. ... \# but Mary doesn't know Sue

c. Every girl knows every other girl

(2) a. The girls are standing on each other

b. ... but Mary is not standing on Sue

c. \# Every girl is standing on every other girl

Dalrymple et al.'s proposal is an account of such contrasts. First, it is assumed that reciprocals are ambiguous. In (1a) the meaning of the reciprocal is

Teresa Galloway and Justin Spence (eds.), SALT VI, 295-310, Ithaca, NY: Cornell University. 
"strong reciprocity" as paraphrased by (1c). In (2a) the meaning of the reciprocal is weaker (let us ignore the details for a while), which allows the sentence to be true also in situations falsifying the strong reciprocity reading (2c). Dalrymple et al. systematically study some possible meanings of reciprocals in various contexts. The second part of their proposal is a principle that determines which reading of the reciprocal is realized in a given sentence. This principle, called the Strongest Meaning Hypothesis, is quoted below.

(3) The Strongest Meaning Hypothesis (SMH): "A reciprocal sentence is interpreted as expressing the logically strongest candidate truth conditions which are not contradicted by known properties of the relation expressed by the reciprocal scope when restricted to the group argument."

Let us exemplify the operation of the SMH in (1a) and (2a). The strongest meaning possible for a reciprocal is strong reciprocity. (1c), the reading this meaning generates for (1a), is compatible with our world (lexical) knowledge about the predicate to know. According to this knowledge (1c) is contingent - it can be true for any number of girls. The SMH claims that the strongest reading possible is also the attested one and therefore in (1a) each other means strong reciprocity. In (2a), however, strong reciprocity would result in the pragmatically implausible paraphrase (2c), which is by necessity false given our knowledge of the predicate to stand on. Consequently, the SMH expects the meaning of (2a) to be weaker, as it is the case.

Dalrymple et al.'s proposal provides, for the first time, an explicit and falsifiable principle that describes the way various interpretations of reciprocals can be obtained. However, there are two reservations of a general nature that I would like to consider: (i) The SMH is introduced as a construction-specific rule for reciprocals. One might expect such a radical connection between lexical knowledge and formal meaning to have a manifestation also in other linguistic contexts. Is it indeed the case? (ii) Can the SMH mechanism be formulated without the unattractive massive ambiguity of reciprocals Dalrymple et al. postulate?

In what follows I will propose affirmative answers to both questions. In fact, it will be shown that they can be viewed as two sides of the same coin.

\section{Plural predicate conjunction}

Cases like (2a) challenge the strong reciprocity treatment of each other. Similarly, a challenge for the traditional Boolean treatment of and can be observed in sentences like (5a). For example, in the situation described by figure 1 below (5a) is true, although a "strong" interpretation as in ( $5 \mathrm{~b})$, obtained using universal quantification and Boolean conjunction, is absurd. (5a) can be roughly paraphrased by: some birds are above the cloud, the others are below it. By contrast, if we consider a minimally different sentence like (4a) the "weak reading" disappears: (4a) is false in the situation of figure 1 . Thus, (4a) reflects approximately the same "strong" statement as (4b), whereas (5a) clearly makes a weaker claim than (5b).

(4) a. The birds are flying above the house and below the cloud.

b. Every bird is flying above the house and below the cloud. 
(5) a. The birds are flying above the cloud and below the cloud.

b. \# Every bird is flying above the cloud and below the cloud.

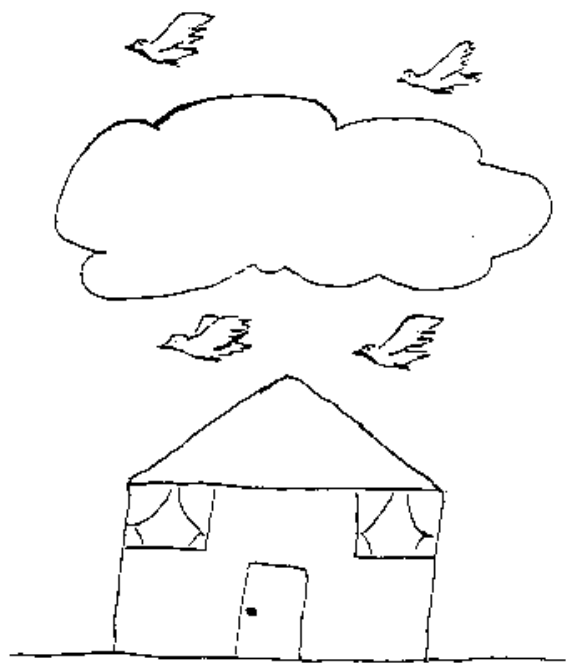

Figure 1: a cloud and a house with birds

Note the parallelism between the contrast (4)-(5) and the contrast (1)-(2). In both pairs the "strong" reading appears when the situations that verify it are possible according to common world knowledge. This is the case in (1a) and (4a). However, when a "strong" interpretation is absurd, as in (2a) and (5a), it is not the actual reading of the sentence. Some principle prefers a contingent weak reading to a stronger but absurd one. In the next section I will propose that in (1)-(2) and in (4)-(5) this principle is the same: the strongest meaning hypothesis.

Contrasts similar to (4)-(5) also exist between (6) and (7) and between (8) and (9) below. Sentence (6) is false in the situation depicted in figure 2 below. This is expected according to a strong reading like every duck is swimming and quacking. In (7), by contrast, such an assertion would contradict our knowledge that ducks cannot both swim and fly at the same time. According to the intuition of the SMH this reading should therefore be weakened, as it is the case: (7) truthfully describes figure 2. Consider now (8) and (9) in a situation with four books. Sentence (8) requires every book to be both old and interesting. In (9), by contrast, two old books and two new ones would suffice to make the sentence true.

(6) The ducks are swimming and quacking.

(7) The ducks are swimming and flying. 


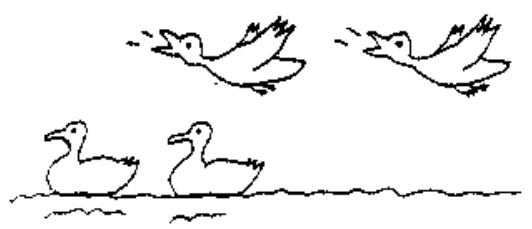

Figure 2: ducks

(8) The books are old and interesting.

(9) The books are old and new.

\section{Extending the SMH}

The examples discussed above support the intuition behind the SMH also in cases of plural predication that do not involve reciprocity: lexical knowledge about predicates can affect the formal meaning of the sentence also with plural predicate conjunction. Let us hypothesize that this effect is a general property of plural predication. We thus modify Dalrymple et al.'s hypothesis as follows:

(10) The (Extended) Strongest Meaning Hypothesis (henceforth SMH): A plural predicate whose meaning is derived from one or more singular predicates is interpreted using the logically strongest truth conditions that are generated from one basic meaning and that are not contradicted by known properties of the singular predicate(s).

This revised formulation of the SMH is obviously too vague. It is not clear what is the "basic meaning" that is subject to weakening and how precisely this process is supposed to work. Let us consider in more detail the proposed implementation of the SMH, deferring some additional technicalities to section 8 .

In order to generate the basic meaning of complex plural predicates we adopt three traditional assumptions about their interpretation:

(i) The meaning of a plural predicate is derived from the meaning of the corresponding singular predicate using universal quantification over members of the group argument. For example:

$$
P_{e t} \rightarrow \mathcal{P}_{(e t) t}=\lambda A_{e t} \cdot \forall x[A(x) \rightarrow P(x)]
$$

(ii) The meaning of a reciprocated plural predicate is derived from the meaning of the corresponding singular $n$-ary predicate using strong reciprocity (SR). For example:

$$
R_{\varepsilon(e t)} \stackrel{S R}{\rightarrow} \mathcal{P}_{(e t) t}=\lambda A_{\epsilon t} . \forall x \in A \forall y \in A[x \neq y \rightarrow R(x, y)]
$$

(iii) Conjunction is Boolean. 
It should be emphasized that the universal quantification schemes assumed in (i) and (ii) above are only approximations to the actual process in plural predication, which is in fact more vague. A sentence like the ducks are quacking is more vague and probably weaker than the sentence every duck is quacking. Similarly, the sentence the men are hitting each other is not equivalent to the sentence every man is hitting every other man. For the sake of the present discussion, however, the universal approximation is sufficient. As in Dalrymple et al. (1994), we will consider only cases with small groups, where such vagueness effects are less prominent.

Assumptions (i)-(iii) are responsible for the derivation of basic meanings of sentences with plural predicates. Weakening effects will be treated using the SMH and will not be conceived as a formal semantic process, but rather as an interaction between formal and lexical semantics. To see how this idea works consider first the basic meaning generated for (1a) and (2a) using strong reciprocity:

(1') $\forall x \in \operatorname{girls}^{\prime} \forall y \in \operatorname{girls}^{\prime}\left[x \neq y \rightarrow \operatorname{know}^{\prime}(x, y)\right]$

(2') $\forall x \in$ girls' $^{\prime} \forall y \in$ girls $^{\prime}\left[x \neq y \rightarrow\right.$ stand_on' $\left.^{\prime}(x, y)\right]$

The operation of the SMH can be intuitively described as follows. Propositions (1') and (2'), instead of being treated as the ultimate readings of (1a) and (2a) (which in the second case would be wrong), are treated as schemes for weakening using the SMH. In order to describe this procedure it is convenient to look at a concrete model as in figure 3(a) below. In this graph a directed arc $g_{1} \rightarrow g_{2}$ corresponds to a (knowing/standing on) relation between girl $g_{1}$ and girl $g_{2}$. The situation in (a) satisfies both (1') and (2'). The crucial point is that while this situation is possible given lexical properties of the predicate to know, it is an impossible state of things with the predicate to stand on. The SMH requires only a graph with a maximal number of arcs that does not contradict the lexical properties of the predicate. Therefore, the SMH predicts that the situation in (a) verifies (1a) and any graph with a smaller number of arcs does not. However, for (2a) the graph in (a) contradicts lexical properties of the predicate to stand on (e.g. anti-symmetry). Thus, also graphs with a smaller number of arcs should do to verify (2a). For example, the graph in figure 3(b) does not contradict lexical properties of to stand on but any additional arc in it would. Thus, according to the SMH this situation verifies $(2 \mathrm{a})$, which is indeed the case. These graphical intuitions will be given a formal correlate in section 8 .

The same intuitions apply in cases of conjunction as (6) and (7). Given the formal assumptions above we generate the following basic meanings for these sentences respectively:

$$
\begin{aligned}
& \forall x \in \mathbf{d u c k s}^{\prime}\left[\mathbf{s w i m}^{\prime}(x) \wedge \text { quack }^{\prime}(x)\right] \\
& \forall x \in \mathbf{d u c k s}^{\prime}\left[\operatorname{swim}^{\prime}(x) \wedge \mathbf{f l y}^{\prime}(x)\right]
\end{aligned}
$$

To make the analogy with (1') and (2') more transparent, note that these formulae are equivalent to $\left(6^{\prime}\right)$ and ( $\left.7^{\prime}\right)$ below respectively:

(6') $\forall x \in$ ducks' $\forall P \in\left\{\right.$ swim' $^{\prime}$ quack' $\}[P(x)]$ 
(a)

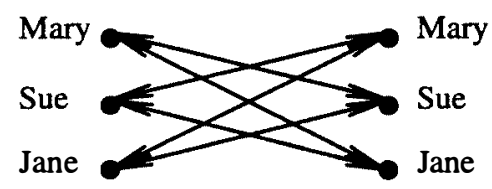

(b)

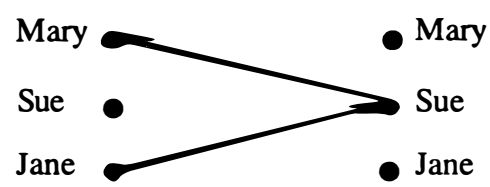

Figure 3: situations verifying (1a) and (2a)

(7') $\forall x \in$ ducks' $\forall P \in\left\{\right.$ swim $^{\prime}$, fly $\left.^{\prime}\right\}[P(x)]$

Thus, in both (1')-(2') and (6')-(7') the propositions involve two universal quantifiers, in the latter case one of them quantifies over predicates. Consider now the situations in figure 4 below. The graph in (a), which attributes every duck both the swimming and quacking properties, is possible given lexical knowledge about these predicates. This situation, therefore, is predicted by the SMH to satisfy (6), whereas a graph with smaller number of arcs does not. In (7), by contrast, the complete graph would contradict world knowledge about the predicates to swim and tofly. The graph in (b) has a maximal number of arcs that does not contradict these properties. Consequently, the SMH predicts that also such a situation verifies (7). This is correct.

(a)

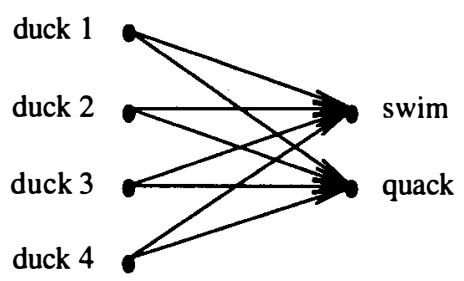

(b)

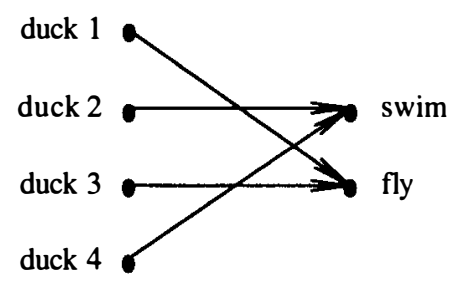

Figure 4: situations verifying (6) and (7)

This account of problematic sentences like (7) has one rather non-standard property. Compare the interpretation of (7) with the following sentential conjunction:

(11) \# The ducks are swimming and the ducks are flying.

a. $\forall x \in$ ducks $^{\prime}\left[\operatorname{swim}^{\prime}(x)\right] \wedge \forall x \in$ ducks' $^{\prime}\left[\mathbf{f l y}^{\prime}(x)\right]$

(11), unlike (7), has a strange flavour: it implies that ducks can swim and fly at the same time. In the treatment proposed here this is so not because of a formal 
semantic mechanism: the basic meanings of (7) and (11) are just the same (7') (=(11a)). However, the difference between (7) and (11) is in the way the SMH applies. The SMH is a principle of plural predication. Therefore, in (11) it applies in each sentential conjunct separately. For these sentential conjuncts the SMH does not have any effect because their meanings do not independently violate any world knowledge of the predicates. Therefore the basic meaning (11a) of (11) is also the attested meaning of the sentence. In (7), by contrast, predicate conjunction compositionally applies before predication and therefore also before the SMH applies. Thus, in (7) it is the basic meaning of the whole sentence that is weakened by the SMH. As we saw, this meaning contradicts lexical properties of the predicates and therefore the SMH has an influence on the attested meaning of the (7). To conclude, the difference between (7) and (11) is not treated at the formal semantic level, but rather as an interference of a semi-pragmatic principle, the $\mathrm{SMH}$, in the predication process.

The intuitive discussion above suggests that the SMH can operate in the same way in cases of reciprocity and in cases of plural predicate conjunction, predicting the semantic similarities observed between the two phenomena. Before moving on to further factual matters, let us briefly discuss a general point where this extension of the $\mathrm{SMH}$ is relevant.

\section{Weakening or strengthening?}

The SMH concept involves semantic weakening of a "default" strong basic meaning. An opposite view, advocated in Langendoen (1978), among many others, is to start with a weak meaning and appeal to pragmatic strengthening in cases like (la). Dalrymple et al. argue that the latter strategy is implausible: cases like (1a) are unlikely to be subject to strengthening via a cancelable pragmatic mechanism like conversational implicature. This is because the strong reciprocity entailment in ( $\mathrm{la}$ ) is not cancelable, as the infelicity of the continuation in (1b) shows. But Mats Rooth (p.c.) raises the following question: why cannot strengthening be obtained by a symmetric principle to the $\mathrm{SMH}$ ? Thus, instead of the weakening strategy of the SMH start with a weak reading and strengthen it using an opposite principle (probably independent of conversational maxims).

The present conception shows a general motivation coming from previous work on conjunction and reciprocals to prefer the SMH to such a strengthening strategy. We saw how the SMH allows us to analyze cases like (5a), (7) and (9) using standard Boolean conjunction. A strengthening analysis of such cases would require to start from a weaker denotation for conjunction, obtained using some definition of "non-Boolean" and. However, while Boolean conjunction was fruitfully given elegant cross-categorial definitions (e.g. in Keenan \& Faltz (1985), Partee \& Rooth (1983)), attempts to generalize non-Boolean definitions of and proved highly problematic (see Winter (1994) on this point). This is a central motivation to pursue the standard Boolean analysis of conjunction also for the treatment of plural predicate conjunction. Consequently, a weakening strategy is to be 
preferred.

Similar considerations also hold with respect to reciprocals as soon as one adopts the decompositional analysis of each other in Heim, Lasnik \& May (1991), where it is proposed to treat the English reciprocal compositionally using the universal expression each and the anaphor other. If this is correct, weakening is preferable also with reciprocals, since their lexical decomposition most easily derives an interpretation using strong reciprocity - universal quantification is obtained by the lexical meaning of each.

\section{Applicability of the SMH and transitive constructions}

Given that the SMH is proposed as a general plurality principle, it is natural to ask: does the SMH apply in all cases of plural predication? As indicated in (10) the answer is negative: the SMH determines only how a predicate that lexically ranges over singular individuals (a "distributive" predicate) can directly apply to plural individuals. Consequently, there are two general cases of plural predication where the SMH does not apply:

1. Predicates that lexically apply to plural individuals ("collective" predicates). For example, predicates like to meet or to lift allow (and in the first case even necessitate) a collective interpretation to the subject argument. Thus, the SMH says nothing about the interpretation of sentences like the boys met or the boys lifted the piano.

2. Cases of predication where some mechanism of distributivity intermediates between the predicate and the plural argument. For example, in order to analyze sentences like (12) correctly, we must assume that the semantics involves universal quantification as in (12a).

(12) Mary and Sue are wearing a dress.

a. $\forall y \in\left\{\mathbf{m}^{\prime}, \mathbf{s}^{\prime}\right\} \exists x\left[\operatorname{dress}^{\prime}(x) \wedge \operatorname{wear}^{\prime}(y, x)\right]$

Without getting into the controversy surrounding the question how distributivity as in (12a) is derived, it is clear that if some grammatical operation derives this interpretation independently of the SMH, then the SMH does not apply in such cases because the correct distributive interpretation involves a universal quantifier mediating between the predicate to wear and the plural argument. Thus, the predicate actually applies to the singular individuals that are being quantified over.

These two general points are important when considering the following question: can the SMH be observed in cases of transitive plural predication that do not involve reciprocity or conjunction? To answer this question effectively, we should first make sure that effects of "collectivity" and "distributivity" as mentioned above do not appear. It is hard to observe any SMH effect in transitive constructions like (13) because the collective interpretation might interfere. 
(13) Mary and Sue lifted the pianos.

Also in sentences like (14) it might be hard to observe the SMH in action because of a distributivity effect sometimes referred to as "partitions": (14) can be true in case a policeman $\mathrm{A}$ arrested a thief $\mathrm{A}$, a policeman $\mathrm{B}$ arrested a thief $\mathrm{B}$, and these policemen and thieves are the ones being referred to by the definite NP's.

(14) The policemen arrested the thieves.

Such partition effects were discussed in Scha (1981) and Schwarzschild (1991) (among others). Schwarzschild proposed to extend some common assumptions about the mechanism of distributivity. Thus, if this idea is correct also (14) is not a convenient test for the SMH.

In order to avoid collective interpretations as in (13), let us concentrate on predicates like to watch, to sit and to give birth, which often resist "togetherness". One way to avoid also partition effects is simply to make the partition reading false: to consider a situation in which no "partition" of the arguments satisfies the predicate. For example, consider sentence (15) in situation (a) of figure 5. There is no way to divide the cats and dogs in the picture into subsets such that in each subset every cat is watching every dog. In such a "no partition" situation the sentence is false: no cat in figure 5(a) is watching dog 3. By contrast, consider sentence (16). In figure 5(b), the same kind of situation as (a), sentence (16) is true although no cat is sitting in basket $3 .^{1}$

(15) The cats are watching the dogs.

(16) The cats are sitting in the baskets.

(a)

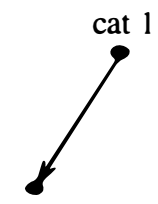

$\operatorname{dog} 1$

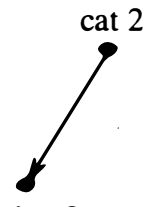

$\operatorname{dog} 2$ $\operatorname{dog} 3$ (b)

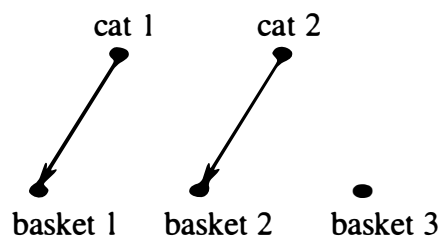

Figure 5: cats, dogs and baskets

Given the SMH in its extended formulation this contrast is expected. A cat can watch more than one dog at the same time and therefore situation (a) in figure 5 does not contain a maximal number of watching relations given the lexical properties of the predicate. In (b) however, any additional "sitting in" relation between a cat and a basket would require one cat to sit in two baskets at the same time. Therefore, according to the SMH this situation is expected to verify (16). 
Another way to check the SMH is to consider cases in which (for reasons that are interesting but need not concern us here) a "partition" interpretation does not seem to appear. Sentence (17) is such a case: in a situation as in figure 6 the sentence seems false. Although this judgement is subtle, notice the contrast with sentence (18), which in the same situation is clearly true.

(17) Mary and Sue saw John, Bill and George.

(18) Mary and Sue gave birth to John, Bill and George.
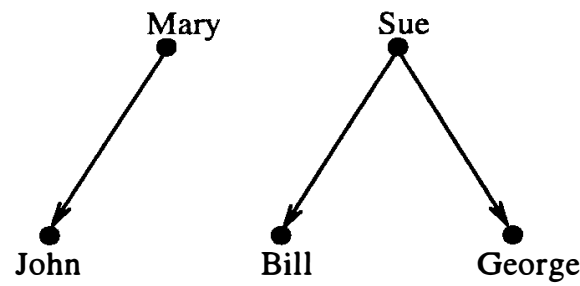

Figure 6: see vs. give birth

The situation in figure 6 does not contain a maximal number of seeing relations, given that it is possible that every woman saw every man. However, it is impossible that two or more women gave birth to the same child and therefore figure 6 contains a maximal number of "giving birth" relations with respect to sentence (18). Consequently, the SMH expects the contrast between (17) and (18) to appear.

\section{SMH interactions}

SMH effects with reciprocals, conjunction and transitive constructions can interact. For example, sentence (19) involves "weak" interpretations for both the conjunction and the reciprocal. To see this consider a situation in which John is sitting on George, who in turn is standing on Bill. Sentence (19) is true in this situation, although there is no boy who is both sitting and standing on another boy, and although neither the sitting nor the standing relation satisfies strong reciprocity with respect to the boys.

(19) The boys are sitting and standing on each other.

The SMH handles such examples correctly. A hyper-graph between couples of boys and sitting/standing relations is depicted in figure 7. Any additional triple-arc connecting two boys and a property would violate properties of to sit on or to stand on. Thus, this situation verifies (19) according to the SMH. In the next section we will see how this is worked out formally.

Sentence (20) is another example for an SMH interaction, this time between transitivity and conjunction: the sentence is true in case the NP these letters refers to 


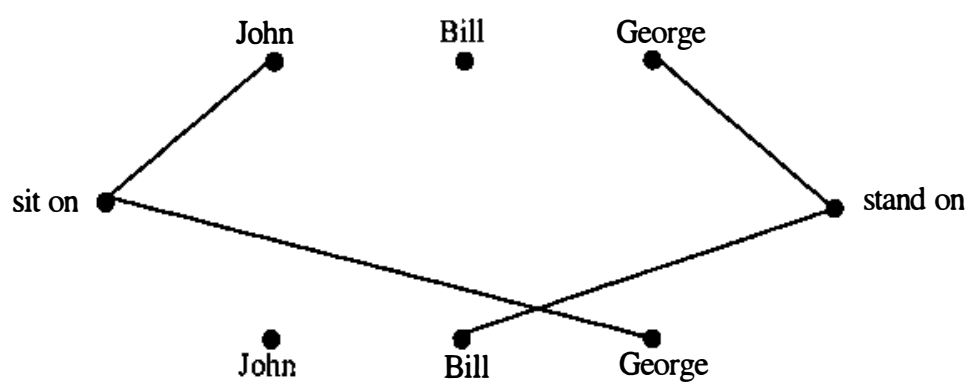

Figure 7: standing and sitting on each other

letters A and B, where letter A was sent by Mary and received by John and letter B was sent by John and received by Mary. Sentence (21), from Sternefeld (to appear), is an example for weakening effects with respect to the reciprocal and the other two arguments of the predicate: (21) is true in the same situation mentioned above for (20). As in (19), the SMH in its extended formulation accounts for these phenomena.

(20) Mary and John sent and received these letters.

(21) Mary and John sent these letters to each other.

An interesting empirical exercise that I did not manage to solve is to try to find cases where the three weakening effects interact.

\section{Formalization}

In formalizing the SMH we first define a normal form for the basic meaning, relative to the tuple of $n$-ary predicates involved. This formula is weakened using world knowledge (meaning postulates) about these predicates, i.e. the conditions on their possible denotations. The underlined notions are defined as follows.

Notation: $\mathcal{N}$ - the natural numbers. For every $s \in \mathcal{N}, \mathcal{N}_{s} \stackrel{\text { def }}{=}\{i \in \mathcal{N}: i \leq s\}$

Definition 1 An $(m, n)$ predicate tuple is an $m$-tuple $\left\langle P_{1}, P_{2}, \ldots, P_{m}\right\rangle$ of $n$-ary predicate constants.

Definition 2 An $(m, n)$ normal universal form relative to an $(m, n)$ predicate tuple $\left\langle P_{1}, P_{2}, \ldots, P_{m}\right\rangle$ is a formula of the form

$\forall i \in \mathcal{N}_{m} \forall x_{1} \in A_{1} \quad \forall x_{2} \in A_{2} \ldots \forall x_{n} \in A_{n}\left[\imath\left(P_{i}, x_{1}, x_{2}, \ldots, x_{n}\right)\right]$

Definition $3 \Theta_{\bar{P}}$, the $(m, n)$ world knowledge about an $(m, n)$ predicate tuple $\bar{P}$ is a subset of $\underbrace{E^{n} \times E^{n} \times \ldots \times E^{n}}_{m}$, where $E$ is the domain of individuals. 
Definition 4 The following formula is the $S M H$ weakening of an $(m, n)$ normal universal form relative to $(m, n)$ world knowledge $\Theta_{\bar{P}}$ :

$\forall\left\langle R_{1}, R_{2}, \ldots R_{m}\right\rangle \in \Theta_{\bar{P}}$

$\left|\left\{\left\langle i, x_{1}, x_{2}, \ldots, x_{n}\right\rangle \in \mathcal{N}_{m} \times A_{1} \times A_{2} \times \ldots \times A_{n}: \psi\left(P_{i}, x_{1}, x_{2}, \ldots, x_{n}\right)\right\}\right| \geq$ $\left|\left\{\left\langle i, x_{1}, x_{2}, \ldots, x_{n}\right\rangle \in \mathcal{N}_{m} \times A_{1} \times A_{2} \times \ldots \times A_{n}: \psi\left(R_{i}, x_{1}, x_{2}, \ldots, x_{n}\right)\right\}\right|$

To exemplify the operation of these definitions consider again sentence (19), which is a rather general case due to the double weakening required in both the reciprocal and the conjunction. Strong reciprocity and Boolean conjunction derive the following basic meaning for $(19)$, in a $(2,2)$ normal universal form:

(22) $\forall i \in\{1,2\} \forall x_{1} \in$ boys' $^{\prime} \forall x_{2} \in$ boys' $^{\prime}\left[x_{1} \neq x_{2} \rightarrow P_{i}\left(x_{1}, x_{2}\right)\right]$

where $\bar{P}=\left\langle\right.$ sit_on' $^{\prime}$, stand_on' ${ }^{\prime}$.

The world knowledge $\Theta_{\bar{P}}$ should reflect the following lexical facts about the meaning of the predicates sit_on' and stand_on': (i) They are mutually exclusive relations: if $x$ is sitting on $y$ then $x$ is not standing on $y$. (ii) Their union describes an acyclic collection of mutually exclusive directed paths. Acyclicity means that if $x_{1}$ is sitting/standing on $x_{2}, x_{2}$ on $x_{3}, \ldots, x_{n-1}$ on $x_{n}$, then $x_{n}$ is neither sitting nor standing on $x_{1}$. The mutual exclusion of paths means that a person cannot sit or stand on more than one person and that two different persons cannot sit or stand on the same person.

If $R_{1}$ and $R_{2}$ are two relations, then the fact that they are mutually exclusive is simply expressed by $R_{1} \cap R_{2}=\emptyset$. We denote the required properties of the relation $R_{1} \cup R_{2}$ by acyclic $\left(R_{1} \cup R_{2}\right)$ and m_ex_path $\left(R_{1} \cup R_{2}\right)$. Acyclicity of a relation $R$ means that its transitive closure is anti-symmetric. Formally:

- The transitive closure of a relation $R, \overline{\operatorname{trans}}(R)$, is a relation recursively defined by:

$$
\begin{aligned}
& \operatorname{trans}_{0}(R)=R \\
& \operatorname{trans}_{i+1}(R)= \\
& \operatorname{trans}_{i}(R) \cup\left\{\langle x, y\rangle: \exists z\left[\langle x, z\rangle \in \operatorname{trans}_{i}(R) \wedge\langle z, y\rangle \in \operatorname{trans}_{i}(R)\right]\right\} \\
& \overline{\operatorname{trans}}(R)=\bigcup_{i=0}^{\infty} \operatorname{trans}_{i}(R)
\end{aligned}
$$

- A relation $R$ is anti-symmetric iff $\forall x \forall y[R(x, y) \rightarrow \neg R(y, x)]$

- A relation $R$ is acyclic iff $\overline{\operatorname{trans}}(R)$ is anti-symmetric.

The requirement about mutual exclusive paths of a relation $R$ is formally defined by:

$\forall x \forall y \forall z[[(R(x, y) \wedge y \neq z) \rightarrow \neg R(x, z)] \wedge[(R(x, y) \wedge x \neq z) \rightarrow \neg R(z, y)]]$

Thus, we define $\Theta_{\bar{P}}$ for $\bar{P}=\left\langle\right.$ sit_on', stand_on' $\left.^{\prime}\right\rangle$ :

$\left\{\left\langle R_{1}, R_{2}\right\rangle \in E^{2} \times E^{2}: R_{1} \cap R_{2}=\emptyset \wedge \operatorname{acyclic}\left(R_{1} \cup R_{2}\right) \wedge\right.$ m_ex_path $\left.\left(R_{1} \cup R_{2}\right)\right\}$

The formula given in (22) above is the normal universal form of the basic meaning of (19). Its SMH weakening is given in (23). 
(23) $\forall\left\langle R_{1}, R_{2}\right\rangle \in \Theta_{\bar{P}}$

$\mid\left\{\left\langle i, x_{1}, x_{2}\right\rangle \in\{1,2\} \times\right.$ boys $^{\prime} \times$ boys' $\left.^{\prime}: x_{1} \neq x_{2} \rightarrow P_{i}\left(x_{1}, x_{2}\right)\right\} \mid \geq$ $\mid\left\{\left\langle i, x_{1}, x_{2}\right\rangle \in\{1,2\} \times\right.$ boys $^{\prime} \times$ boys $\left.^{\prime}: x_{1} \neq x_{2} \rightarrow R_{i}\left(x_{1}, x_{2}\right)\right\} \mid$

In words: for any relations $R_{1}, R_{2}$ that are possible denotations of sit_on' and stand_on' respectively, the total number of pairs of different boys $x_{1}, x_{2}$ such that sit_on' $\left(x_{1}, x_{2}\right)$, plus the total number of pairs of different boys $x_{1}, x_{2}$ such that stand_on' $\left(x_{1}, x_{2}\right)$ is greater or equal to the similar sum with $R_{1}$ and $R_{2}$.

Let us observe now that the situation in figure 7 satisfies (23). Note first that in this situation

$\mid\left\{\left\langle i, x_{1}, x_{2}\right\rangle \in\{1,2\} \times\right.$ boys $^{\prime} \times$ boys' $\left.^{\prime}: x_{1} \neq x_{2} \rightarrow P_{i}\left(x_{1}, x_{2}\right)\right\} \mid=8$

This is because the following tuples $\left\langle i, x_{1}, x_{2}\right\rangle$ in $\{1,2\} \times$ boys' $^{\prime} \times$ boys $^{\prime}$ are the ones for which the formula $x_{1} \neq x_{2} \rightarrow P_{i}\left(x_{1}, x_{2}\right)$ holds:

$\left\langle 1, \mathbf{j}^{\prime}, \mathbf{j}^{\prime}\right\rangle\left\langle 2, \mathbf{j}^{\prime}, \mathbf{j}^{\prime}\right\rangle\left\langle 1, \mathbf{b}^{\prime}, \mathbf{b}^{\prime}\right\rangle\left\langle 2, \mathbf{b}^{\prime}, \mathbf{b}^{\prime}\right\rangle\left\langle 1, \mathbf{g}^{\prime}, \mathbf{g}^{\prime}\right\rangle\left\langle 2, \mathbf{g}^{\prime}, \mathbf{g}^{\prime}\right\rangle\left\langle 1, \mathbf{j}^{\prime}, \mathbf{g}^{\prime}\right\rangle\left\langle 2, \mathbf{g}^{\prime}, \mathbf{b}^{\prime}\right\rangle$

Let us denote:

$s\left(R_{1}, R_{2}\right)=\mid\left\{\left\langle i, x_{1}, x_{2}\right\rangle \in\{1,2\} \times\right.$ boys $^{\prime} \times$ boys $\left.^{\prime}: x_{1} \neq x_{2} \rightarrow R_{i}\left(x_{1}, x_{2}\right)\right\} \mid$

We want to show: $\forall\left\langle R_{1}, R_{2}\right\rangle \in \Theta_{\bar{P}}\left[8 \geq s\left(R_{1}, R_{2}\right)\right]$

The proof is by constructing $R_{1}, R_{2}$ in $\Theta_{\bar{P}}$ with maximal $s\left(R_{1}, R_{2}\right)$. Without loss of generality, assume $R_{1}\left(\mathbf{j}^{\prime}, \mathbf{g}^{\prime}\right)$. Let us denote $R_{i}^{\mathbf{b}}=R_{i} \cap\left(\right.$ boys $^{\prime} \times$ boys $\left.^{\prime}\right)$. It is not hard to show by enumeration of cases that one of the following conditions must hold if $R_{1}$ and $R_{2}$ satisfy $s\left(R_{1}, R_{2}\right) \geq 8$ and $\left\langle R_{1}, R_{2}\right\rangle \in \Theta_{\bar{P}}$ :

1. $R_{1}^{\mathbf{b}}=\left\{\left\langle\mathbf{j}^{\prime}, \mathbf{g}^{\prime}\right\rangle\right\}, \quad R_{2}^{\mathbf{b}}=\left\{\left\langle\mathbf{g}^{\prime}, \mathbf{b}^{\prime}\right\rangle\right\}$

2. $R_{1}^{\mathbf{b}}=\left\{\left\langle\mathbf{j}^{\prime}, \mathbf{g}^{\prime}\right\rangle,\left\langle\mathbf{g}^{\prime}, \mathbf{b}^{\prime}\right\rangle\right\}, \quad R_{2}^{\mathbf{b}}=\emptyset$

3. $R_{1}^{\mathbf{b}}=\left\{\left\langle\mathbf{j}^{\prime}, \mathbf{g}^{\prime}\right\rangle\right\}, \quad R_{2}^{\mathbf{b}}=\left\{\left\langle\mathbf{b}^{\prime}, \mathbf{j}^{\prime}\right\rangle\right\}$

4. $R_{1}^{\mathbf{b}}=\left\{\left\langle\mathbf{j}^{\prime}, \mathbf{g}^{\prime}\right\rangle,\left\langle\mathbf{b}^{\prime}, \mathbf{j}^{\prime}\right\rangle\right\}, \quad R_{2}^{\mathbf{b}}=\emptyset$

In Other words: given that $R_{1}\left(\mathbf{j}^{\prime}, \mathbf{g}^{\prime}\right)$ holds, in order for $R_{1}$ and $R_{2}$ to satisfy $s\left(R_{1}, R_{2}\right) \geq 8$ without satisfying one of the four conditions above, they must violate the condition $\left\langle R_{1}, R_{2}\right\rangle \in \Theta_{\bar{P}}$

But for the four possibilities above $s\left(R_{1}, R_{2}\right)=8$, which means that (23) is true.

\section{Loose ends}

There are some cases in which the strongest meaning hypothesis seems to be too strong. Namely, weakening occurs although it is not supposed to according to the SMH. Consider the following example from Philip (1996):

(24) The boys are tickling each other.

In the situation of figure 8(a) below (24) is true although the SMH expects it to be false: it is possible for every boy to tickle both other boys in the picture. 
(a)

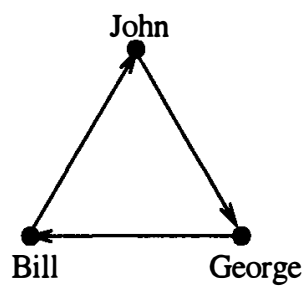

(b)

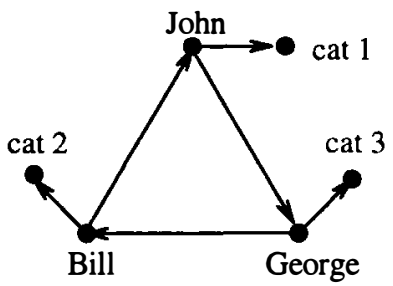

Figure 8: boys tickling each other

The reason for this potential counter-example to the SMH might be some gap in our world knowledge about predicates like to tickle: although it can happen that a boy tickles more than one object, this might not be the default assumption about the predicate. In principle, it might be that the lexical knowledge about the predicate does require "uniqueness" of an object tickled by a person, and this would allow the SMH to account for the interpretation of (24). However, it is completely possible for John to tickle both George and Bill at the same time. Therefore, we must assume that the "uniqueness" default can be overridden: it is not as robust as the lexical knowledge we considered with predicates like to stand on or to give birth to. This immediately predicts that whenever the default assumption is overridden also the SMH ignores it. For example, in the situation in figure 8(b) every boy is tickling also a cat, in addition to the boy he is already tickling in (a). Consequently, we expect (24) to be interpreted differently in situation (b) than it is in (a): the sentence is expected to be false in (b) because in this case it is evident that every boy is not tickling the maximal number of boys he potentially can. Whether this prediction is borne out is not too clear. It seems to me that in such delicate pragmatic matters introspection is not enough and careful experimental research is needed.

There is also an opposite problem. In certain cases the SMH expects weakening to occur although actually it does not. Consider the following examples:

(25) \# Mary and Sue gave birth to each other. (after Sauerland (1994))

(26) \# Mary and Sue gave birth to John. (after Edwin Williams (p.c.))

In sentence (25) the SMH expects a weakened interpretation: either Mary gave Birth to Sue or Sue gave birth to Mary. This is clearly not the case because the oddness of the sentence indicates that it is a case of strong reciprocity: the implausible claim that Mary gave birth to Sue and vice versa. The same with (26): since it is impossible that two women gave birth to the same child the SMH expects (26) to have a weak, plausible, interpretation: either Mary or Sue gave birth to John. This is incorrect. It seems that although the predicate to give birth can allow some weakening effects (cf. (18)), there is a "lower bound" to the weakening that can take 
place: each member of the group argument should take part in at least one "giving birth" relation. Why this is so is an open question.

\section{Conclusion}

The strongest meaning hypothesis makes a non-standard connection between lexical semantics and formal semantics. It implies that the seemingly unsystematic semantic behaviour of predication with plurals is a manifestation of an incomplete formal semantics: some lexical semantic information is required in order to compute the meaning of a plural predicate. In this paper I tried to show that this perspective on Dalrymple et al.'s study of reciprocals can reveal systematicity also in other cases of plural predication. No less important for the objectives of the present enterprise is the possibility it opens to avoid lexical ambiguities or foundational complications in the formal semantic apparatus. Contrary to common opinions, the minimalistic and elegant assumptions of Boolean semantics might prove to be linguistically enlightening also for the shady domain of plurality.

\section{Endnotes}

* Thanks to the participants of SALT6 and to the audiences at lectures given in OTS and UCLA for helpful comments. Especially, I am grateful to Ed Keenan, Bill Philip, Uli Sauerland, Tim Stowell, Anna Szabolcsi and Henk Verkuyl. Thanks to Yael Seggev for the drawings. Financial support was provided by the Dutch Organization for Scientific Research (NWO) and by the Netherlands Graduate School of Linguistics (LOT).

1. Uli Sauerland (p.c.) mentions a similar contrast that was independently observed by Roger Schwarzschild.

\section{References}

Dalrymple, M., M. Kanazawa, S. Mchombo and S. Peters (1994), "What do reciprocals mean?", in Proceedings of SALT4.

Heim, I., H. Lasnik and R. May (1991), "Reciprocity and plurality", Linguistic Inquiry 22: 63-101.

Keenan, E., and L. Faltz (1985), Boolean Semantics for Natural Language, Reidel. Langendoen, D.T. (1978), "The logic of reciprocity", Linguistic Inquiry 9: 177-197.

Partee, B., and M. Rooth (1983), "Generalized conjunction and type ambiguity”, in R. Bauerle et al.(eds.), Meaning, Use and Interpretation of Language, De Gruyter.

Philip, W. (1996), “Children who know each other”, ms., OTS, Utrecht University.

Sauerland, U. (1994), "Codistributivity and reciprocals", in Proceedings of WECOL94.

Scha, R. (1981), "Distributive, collective and cumulative quantification", in J. Groenendijk, M. Stokhof and T.M.V. Janssen(eds.), Formal Methods in the Study 
of Language, Amsterdam.

Schwarzschild, R.S. (1991), On the Meaning of Definite Plural Noun Phrases, Ph.D. diss., UMASS.

Sternefeld, W. (to appear), "Reciprocity and cumulative predication", in F. Hamm and E. Hinrichs(eds.), Plurality and Quantification, Kluwer.

Winter, Y. (1994), "A unified semantic treatment of singular NP coordination", ms., OTS. To appear in Linguistics and Philosophy.

OTS, Utrecht University, Trans 10, 3512 JK Utrecht, The Netherlands e-mail: yoad.winter@let.ruu.nl 